\title{
Metrology, essential to trade, industry and society
}

\author{
Robert Kaarls
}

Received: 4 July 2007 / Accepted: 5 July 2007 / Published online: 26 July 2007

(C) Springer-Verlag 2007

Keywords Metrology - Economic and societal impact . Technical barriers to trade $\cdot$ Proficiency testing .

CRMs · CIPM MRA $\cdot$ CCQM

Internal markets as well as the globalization of trade, industry and society require comparability of calibration, measurement and test results through traceability to the International System of Units (SI), which represents the coherent and long-term stable fixed anchor points in measurement. Internal markets, trade and accreditation agreements also require that non-tariff barriers to trade are removed. The removal of Technical Barriers to Trade (TBT) and implementation of Sanitary and Phyto-Sanitary (SPS) measures should lead to a situation where 'once measured or tested, everywhere accepted'. To achieve this situation, a credible, transparent metrology agreement needs to be implemented under the Inter-Governmental Treaty of the Metre Convention. The need for a reliable world metrology system is driven not only by trade requirements but equally by societal requirements.

Presented at the 3rd International Conference on Metrology, November 2006, Tel Aviv, Israel.

Electronic supplementary material The online version of this article (doi:10.1007/s00769-007-0301-6) contains supplementary material, which is available to authorized users.

R. Kaarls $(\bowtie)$

CIPM, CCQM, Klaverwydenstraat 13,

2381 VX Zoeterwoude, The Netherlands

e-mail: rkaarls@euronet.nl
Improvement of the quality of life is highly served by reliable, traceable and more accurate measurements, particularly in areas such as health care, food safety and nutritional content, environmental and pollution control and climate change, forensics and security. In addition, credible 'soft/perceptive' measurements, such as smell, taste, colour, glance, form, among others, will contribute to the quality of life.

Sustainable competitiveness and innovation require accurate, traceable measurement results, not only in the 'classical' field of physical measurements, but also in the fields of chemical analysis, advanced materials and material properties and in new fields like nano-metrology and biotechnology. These new areas of metrology also require the development of new measurement technologies. The wide application of information technology, in the form of fully automated, remote-controlled and monitored measurement systems, also requires new innovative approaches.

In as much that accurate, comparable measurements are essential for a fair and safe society, it is evident that the authorities have formulated and are increasingly formulating regulations that contain requirements with respect to traceability and measurement uncertainty.

The economic interest in good measurements is well demonstrated by the fact that global trade in commodities amounts to more than 12 trillion USD, of which $80 \%$ is affected by standards and regulation. The compliance costs are estimated to be about $10 \%$ of the production costs. The global markets of clinical chemistry and laboratory medicine and pharmaceuticals have a value of some 300 billion USD per year. Annual savings as a consequence of comparable, more accurate measurement results with a smaller measurement uncertainty will easily amount up to many billions of U.S. dollars. 
The economy of developing countries is largely dependent on the export of food products (40\% of Gross Domestic Product) and raw materials (also $40 \%$ of GDP).

That there is a need to improve the national metrological infrastructures in many countries is continuously being demonstrated by the results of inter-comparisons, for example by those of many IMEP (International Measurement Evaluation Programme) studies carried out by the Institute of Reference Materials and Measurements (IRMM) [1]. Inter-laboratory comparisons, like those carried out by the BAM (German Federal Institute for Materials Research and Testing) and by FAPAS (UK Food Analysis Performance Assessment Scheme) in the area of food testing, show the same unsatisfactory results. The measurement results of participating laboratories show a wide dispersal - while, in many cases, small uncertainties are claimed. Similarly, there does not seem to be a clear relation between accreditation and good measurement results. The lead in wine comparison carried out by the IRMM is a typical example. However, one should be able to perform better, as is demonstrated by the results in the associated CCQM pilot study comparison.

A particular example is the area of metrology in chemistry, where issues still have to be addressed, such as a full understanding and description of the measurand and the full understanding of the measurement process and its uncertainty components. Although enormous progress has been made over the last 10 years in terms of establishing comparability of measurement results in chemistry and, in addition, it has been demonstrated that comparability can be also realized for more complex molecules and chemical matrices, still more study is required.

There is a great demand for adequate Certified Reference Materials (CRMs) as the means of disseminating traceability to the measurement and test laboratories. For the same reasons, it would be highly desirable that Proficiency Tests are carried out with an assigned traceable reference value. The FAPAS example of tin in tomato puree demonstrates the dangers of drawing conclusions on the basis of consensus values. Unfortunately, in many proficiency testing (PT) schemes no assigned reference value is used. Another concern is that a considerable discrepancy exists between CRMs needed in the scope of PT schemes and CRMs that are commercially available on the market.

The Inter-Governmental Treaty of the Metre Convention aims to establish and maintain a unique, long-term stable, global measurement system which defines measurement units and measurement standards. The organs of the Treaty - the International Committee for Weights and Measures (CIPM), its scientific Consultative Committees (CCs) and the International Bureau BIPM in Sèvres (Paris), France are in charge of disseminating traceability, facilitating international cooperation, organizing comparisons, representing the global metrological community and liaising with other inter-governmental and international organizations with an interest in metrology. Global mutual recognition of national measurement standards and of calibration and measurement certificates issued by the National Metrology Institutes (NMIs) and other designated institutes constitute an essential part of the activities under the Metre Convention.

The CIPM Mutual Recognition Arrangement (CIPM MRA) is based on the results of Key Comparisons and the implementation and review of quality systems in accordance with ISO/IEC 17025 and the ISO Guide 34 (for Certified Reference Materials). The reviewed calibration and measurement capabilities of the NMIs and other designated institutes are published by the International Bureau for Weights and Measures (BIPM) in the KCDB (Key Comparison Data Base) [2].

The Consultative Committee for Amount of Substance (CCQM), which is the Consultative Committee of the CIPM in charge of metrology in chemistry, and the CCQM Working Groups realize global comparability through traceability by organizing Key Comparisons and Studies. For the results of these Key Comparisons and Studies, see the BIPM website [2]. By liaising with a large number of other inter-governmental and international organizations with interest in reliable and traceable measurements, the CCQM determines the right priorities and is able to link the global metrological network with the reference and 'field' laboratories in the different sectors; in doing so, it establishes the basis for international recognition and acceptance of measurement and test results produced by the sectorrelated laboratories. Areas of metrological priority are the food sector, the health care sector and the environmental (pollution and climate change) sector.

International cooperation between the CIPM/BIPM/ CCQM and other inter-governmental and international organizations is developing very quickly. The food sector provides one such example with cooperation between the Codex Alimentarius Commission, the Inter-Agency Meeting (IAM), the Association of Official Analytical Chemists-International (AOAC-I) and the World Health Organization (WHO) as well as with the genetically modified organisms (GMO) industry and testing laboratories. Other examples include the health care sector, with the WHO, IFCC (International Federation of Clinical Chemistry and Laboratory Medicine) and JCTLM (Joint Committee on Traceability in Laboratory Medicine), and the area of environmental measurements and climate change, with the WMO (World Meteorological Organization) and others. Further liaisons have been established with the World Anti-Doping Agency (WADA), the Forensics laboratory community, the Pharmacopeia, International 
Laboratory Accreditation Organization (ILAC), International Standardization Organization (ISO), regulators and sector-specific industry and calibration and testing laboratory associations.

An interesting spin-off of the CIPM MRA and the activities carried out under the CCQM is the creation of the JCTLM. The JCTLM is composed of representatives from the BIPM, IFCC and ILAC, with full support of all other stakeholders. The JCTLM is currently publishing lists of reliable, traceable reference materials and reference methods of a higher order that comply with the requirements of the EU In-Vitro Diagnostics directive. For more details, see the website of the BIPM [2] or the IFCC [3].

The lack of a reliable national infrastructure in the relatively new field of metrology in chemistry hinders an easy and complete dissemination of traceability to the field laboratories. The accreditation of a second level of calibration laboratories that are in charge of disseminating traceability to the 'field' laboratories, is well established in the area of 'classical' physical measurements. However, such a network is largely lacking in the area of chemical measurements. The development of a second layer of accredited "calibration" laboratories on the basis of ISO/
IEC 17025 and accredited CRM producers on the basis of ISO/IEC 17025 and ISO Guide 34 should be fostered.

Accredited organizations act as secondary "reference" laboratories, disseminating traceability to the field laboratories, while the reference laboratories themselves obtain their traceability from NMIs and other designated institutes that act as a NMI in a defined area of metrology. The accreditation of PT providers on the basis of ISO Guide 43, now being under review as a new ISO standard 17043, will help to select reliable PT providers, which hopefully will work with assigned reference values in their schemes.

The CIPM MRA and the ILAC Arrangement are fully complementary and, when implemented in all areas of measurement, constitute a reliable network of traceability on behalf of trade, industry and society.

\section{References}

1. www.imep.ws

2. www.bipm.org

3. www.ifcc.org 